\title{
Electrophysiological Investigation of Episodic Encoding in Schizophrenia
}

\author{
Michael Chih Chien Kuo* \\ School of Medical and Health Sciences, Tung Wah College, Hong Kong, China \\ *Correspondence to: Michael C. C. Kuo, Ph.D; Tung Wah College, School of Medical and Health Sciences, 98 \\ Shantung Street, Mongkok, Kowloon, Hong Kong. Tel: +852 3468 6656; Fax: +852 3753 2106; Email: michaelkuo@twc. \\ edu.hk
}

Published online March 15, 2018

\section{Dear Editor,}

Besides psychiatric symptoms, deficits in episodic memory are frequently experienced in people with schizophrenia. Memory involves encoding, storage, and retrieval. While all three stages are important, information has to be accurately encoded before they can be stored and retrieved. ${ }^{1}$ Even though there have been reports that described changes in brain activation while performing an episodic encoding task in schizophrenia, very little is known about the time dimension of these cognitive processes. The event-related potential (ERP) technique is well suited for the temporal and precise measurement of the brain electrical activities resulting from cognitive processes because of its resolution at millisecond scale. Previous ERP studies that have been conducted in schizophrenia tended to focus on preattentive information processing, ${ }^{2}$ social cognition ${ }^{3}$ or memory retrieval. ${ }^{4,5}$ To my knowledge, only two recent investigations $^{6,7}$ have examined temporal aspect of episodic encoding. Green et $\mathrm{al}^{7}$ focused on both early perceptual and late encoding processes. Their participants performed semantic encoding by determining whether words displayed on a computer screen gave a pleasant or unpleasant feeling. As evidenced by their results, early encoding processes were unchanged in schizophrenia while changes in later (and higher order) processes contributed to their deficits in episodic encoding. Battal Merlet et $\mathrm{al}^{6}$ examined semantic coherence effect during episodic encoding in schizophrenia using a N400 paradigm. Their N400 results indicated that people with schizophrenia could take external semantic cues and use them to improve memory performance. However, they were unable to process implicit inter-item relationships. These two studies may be the beginning of potential a new line of research in schizophrenia. Future related studies might consider the following:

1. Including a baseline condition where participants are not instructed to use a specific encoding method. To convincingly claim whether and how much different methods of encoding might benefit people with schizophrenia or not and examine the cognitive processes that lead to changes in memory performance, this condition would be necessary.

2. If a subsequent memory paradigm is used as in Green et al, ${ }^{7}$ participants will need to be trained and prompted of the proper task-taking procedure to reduce the likelihood of retrieval strategies affecting encoding results. ${ }^{8}$ In addition, task design should allow differentiation of confidently remembered trials from less confidently remembered trials. As more variabilities in cognitive processes are likely to be observed in schizophrenia, this procedure increases the possibility of finding the effects intended.

3. Performing source analysis to identify neural substrates associated with the cognitive processes. This should also permit some comparisons with reports that used the functional magnetic resonance imaging technique.

4. Using a larger sample size to allow comparisons of differential effects of encoding methods across subgroups, such as years after disease onset or severity of psychiatric symptoms.

It is hopeful that as more knowledge about the encoding processes of people with schizophrenia is gained, new intervention strategies for their memory remediation may be developed.

Conflict of Interest Disclosures

The authors declare that they have no conflict of interests.

Ethical Statement

Not applicable.

Financial Disclosure

The author has no financial interests related to the material in the manuscript. 
References

1. Chih Chien Kuo M. Event-Related Potential Investigation of the Mechanisms of Memory Encoding in Schizophrenia. Arch Neurosci. 2018;5(1):e64776. doi: 10.5812/ archneurosci.64776.

2. Lee M, Sehatpour P, Hoptman MJ, Lakatos P, Dias EC, Kantrowitz JT, et al. Neural mechanisms of mismatch negativity dysfunction in schizophrenia. Mol Psychiatry. 2017;22(11):1585-93. doi: 10.1038/mp.2017.3.

3. Brenner CA, Rumak SP, Burns AMN. Facial emotion memory in schizophrenia: From encoding to maintenance-related EEG. Clin Neurophysiol. 2016;127(2):1366-73. doi: 10.1016/j. clinph.2015.10.061.

4. Guillaume F, Guillem F, Tiberghien G, Stip E. Mismatched expressions decrease face recognition and corresponding ERP old/new effects in schizophrenia. Neuropsychology. 2012;26(5):568-77. doi: 10.1037/a0028924.

5. Guillaume F, Stip E, Tiberghien G. About the nature of contextual impairments revealed by FN400 abnormalities in schizophrenia. Front Hum Neurosci. 2013;7:60. doi: 10.3389/fnhum.2013.00060.

6. Battal Merlet L, Blanchet A, Lockman H, Kostova M. An Event Related Potentials Study of Semantic Coherence Effect during Episodic Encoding in Schizophrenia Patients. Schizophr Res Treatment. 2018;2018:8501973. doi: 10.1155/2018/8501973.

7. Green AE, Fitzgerald PB, Johnston PJ, Nathan PJ, Kulkarni J, Croft RJ. Evidence for a differential contribution of early perceptual and late cognitive processes during encoding to episodic memory impairment in schizophrenia. World J Biol Psychiatry. 2017:18(5):369-81. doi: 10.1080/15622975.2016.1208839.

8. Bridger EK, Wilding EL. Requirements at retrieval modulate subsequent memory effects: An event-related potential study. Cogn Neurosci. 2010;1(4):254-60. doi: 10.1080/17588928.2010.484882.

Citation: Kuo MCC. Electrophysiological investigation of episodic encoding in schizophrenia. Int Clin Neurosci J. 2018;5(1):1-2. doi:10.15171/icnj.2018.01 